\title{
A Cooperation Model and Demand-Oriented ICT Infrastructure for SME Development and Production Networks in the Field of Microsystem Technology
}

\author{
Markus Dickerhof \\ Karlsruhe Institute of Technology \\ Institute for Applied Computer Science \\ Hermann v. Helmholtz Platz 1 \\ D 76344 Eggenstein Leopoldshafen \\ Tel.: +497247825754 \\ Fax. +497247825786 \\ markus.dickerhofakit.edu
}

\begin{abstract}
Keywords: Collaborative engineering, new collaboration models Distributed and collaborative networks Readiness for participation in Virtual Enterprises.
\end{abstract}

\section{Introduction}

Like no other industry, until today microsystems technology is dominated by a few, large companies covering the whole development and production chain. Besides limitations coming from huge costs for equipment and manufacturing (especially in the field of Si-Microsystem technology) one of the reasons for the weak position of Small Medium Enterprises (SME) in this branch refers to organizational issues, arising from the specific surrounding conditions in this highly interdisciplinary and knowledge intensive field. Especially the smaller SME's lack of sufficient human resources and an effective management of cross company knowledge about complex Microsystems development to cover all aspects of a complex and parallel product and process development as it is inherent in the one product- one process paradigm of MST.

Since most of the Microsystems technologies until today haven't reached an almost mature state it near at hand, that there is a demand for approaches, offering the chance to effectively handle the riscs of a MST product development - which basically is not a technical but an organizational problem. This aspect gains more and more importance when the number of companies involved in such a process development increases. If SME networks seriously want to compete with larger companies, new approaches on how to handle a distributed knowledge intensive product development process need to be found.

\section{A New Concept for Small Medium Enterprise Cooperations in the Field of Microsystems Technology}

The starting point for bases on the perception, that neither the type nor the intensity of a cooperation can be seen as a static process. Much more the aspects mentioned before 
depend on the intensity of the cooperation which was identified as a function of a chronological network development and of the intended purpose of the cooperation, both significantly influencing the choice of appropriate tools and methodologies.

Figure 1 gives a schematic overview of a classification scheme, allowing for the arrangement of organizations in four types of cooperation intensity. Each of these type has been identified to be existing in / of relevance for the Microsystems community. While the weakest type of cooperation, the market place (e.g. IVAM) primarily bases on the idea of a non-binding common platform for marketing, cooperation type four, the so called "Clan-Cooperation", represents the strongest one [Picot93]. A modified version of this approach has been identified as the most intensive and probably the most promising one for innovative MST companies in a German national funded project [MWF03].

The MST-"Clan-Cooperation" idea bases on the assumption, that in a MST cooperation the partners do not only have to share their production capacity in terms of what was called "virtual enterprise" in the late 90's [VIRT09]. Furthermore the also share intensively their production and application knowledge enabling them to offer "one-stop-shop" services to their customers, e.g. as an OEM solution provider.

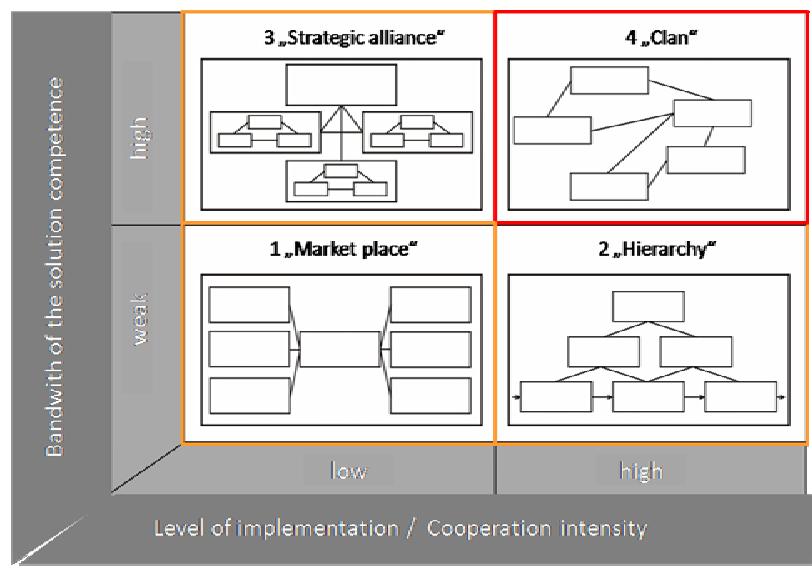

Fig. 1. SME-cooperation types in microsystems technology (modified model acc. to [PICO93])

Looking at the clan-cooperation model one can imagine that the inherent flexibility of this model provides significant advantages in the MST product development phase, but it is also clear, that the non-binding structure of the clan model finally is not the appropriate approach for a later production either in a small or in a medium or large scale. In this phase of the product development cycle the organizational framework certainly will result in a type of "general suppliership", conducted one of the partners out of the consortium. This general supplkier takes the lead in the consortium and integrated all other partner as subcontractors, probably using technologies introduced in the Virtual Enterprise context [MUEL99]. Hence, as soon as the MST product life cycle passes from the development to the production state, its differs no longer very much from other macro world production consortiums, as it has at least to adress the same requirements on quality, reliability, documentation, 
and productivity as other macro-scale components, integrated by large companies into their macro scale systems.

\section{Demand-Oriented Software System Support for MST Development Process}

The following perceptions have been made while a detailed analysis and discussion of the Clan cooperation model [DICK08]:

- Organisation and support tools must be flexible enough to support information sharing and the need for communication in an appropriate manner.

- In parallel to the rising amount of projects, and with the rising degree of integration of the network, the coordination should select appropriate tools in a demand-oriented manner. This means that in most cases not the most ambitious technological are the best solutions. Furthermore the ICT Infrastructure needs to be scalable and fittable. However the state of the art R\&D projects in this field still focusses on the development of information and communication environments for mature organisation in the supply chain or large company context and do not yield appropriate solutions for SME networks.

- Information management, content management, shared workspace environments, videoconferencing can bring - due to the reduced amount of technical meetings - real value to all partners at a very early stage of a cooperation.

Based on these perceptions five basic ICT-requirements have been identified for the clan cooperation. A customized ICT infrastructure for a joint development and production in Microsystems technologies has

- to address the different phases of an application / production development.

- to support the heterogeneous infrastructures in the participating companies.

- to be flexible enough to deal with the different intensity of information exchange referring to the different phases of the cooperation life cycle. While the knowledge sharing intensity in a market place rests almost on the same level or raises up and decreases in a temporally limited cooperation like the virtual enterprise, it may continuously increase in a strategic alliance or a clan cooperation (Fig. 2).

- to address the specific constraints of the MST, resulting out of the still insufficient maturity of the technology itself. In most cases, the partner will not only have to deal with a cross company application development but also with a cross company production process development / adoption of established processes.

- to consider, that standardization in the information exchange on shop floor level (standardization of transport equipment) as well as on the logistic level is very weak in this community and in most cases belongs to the requirements of an end customer (if there exist one).

An effective and user centered infrastructure has to deal with all requirements mentioned above, if it shall not fail in terms of appropriateness and user friendliness. 


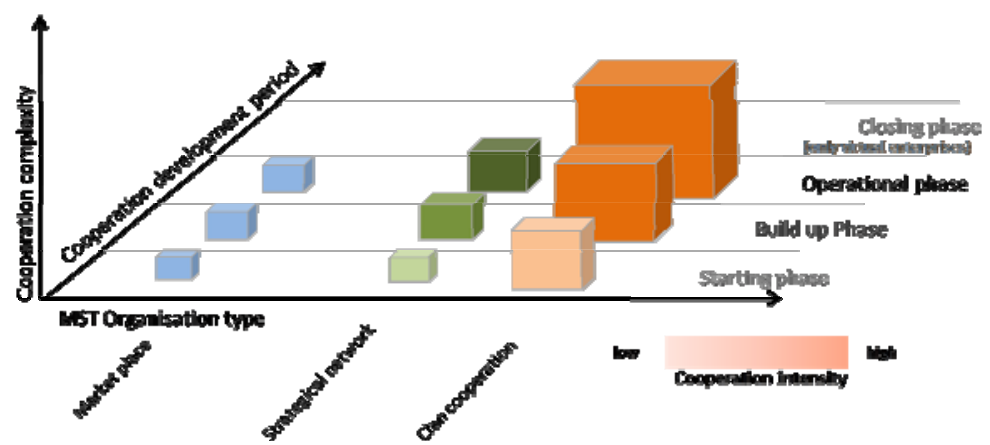

Fig. 2. Timely development of the cooperation intensity in typical SME networks related to the microsystems branche

\section{Software Support for the MST Development Process on Organizational Level}

In Section two came out, that for the production phase and from the organizational point of view a MST-SME cooperation acts similar to a macro production network, using almost the same tools for monitoring and control. Due to this work on MST specific software aspects will sooner or later focus on the technological aspects of assisting distributed teams while the knowledge intensive product development process. The following chapter summarizes a solution concept, supporting the "Open solution-paradigm".

Supporting collaborative teams through workflow technologies

Coming from the above mentioned "one product-one process paradigm" MST- SME cooperation -in contrary to "macro world"-production networks have to deal with a parallel process and product development which basically can be described as an "open-solution” process (Fig. 3).

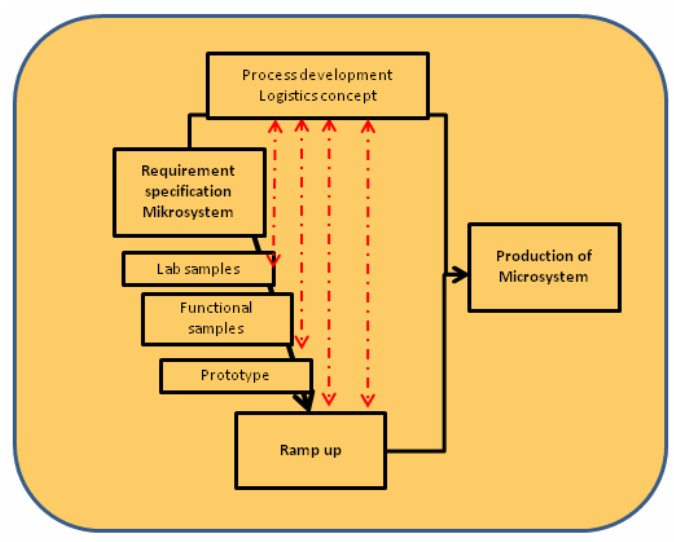

Fig. 3. Parallel process and product development in MST 
"Open-Solution" process in this context means, that the final solution strategies for either the product- or the related production development process are not clear at an early phase of MST component developments.

This implies that the product development process itself can't be finally specified from the beginning on. Other the hand side it does not mean that single steps in the development can't be well specified, executed and analyzed which leads one to the conclusion that MST development processes need to be planned in a way that allows for a break down in small manageable pieces.

An appropriate solution path to handle such "solution open" developments is to describe the development steps indirectly through the definition of milestones for each phase that have to be achieved after a certain time or number of iterations. The milestones again can be clustered in such a way, that a set of milestones describe specific development phases, where at the end both, the customer and the project consortium, have to decide whether to continue or not. Anyway the solution-open problem still remains unsolved at this point.

For the modeling of this specific constraint we modified the Spiral-model, introduced by B. W. Boehm [BOEHM88]. The spiral model was introduced in 1988 and is until today one of the major software development models for handling the iterative software development process in an efficient way. We adopted the model to the needs of MST development, allowing us to describe each process by four basic sections, valid for all phases of the MST product development process: the brainstorming phase, the elaboration phase, the internal review and the external review (Fig. 4, Sections A-D). If the single development step was accomplished with satisfying results the development process continues to the next phase. If not, the same sequence has to be processed again.

In a next step we modeled each project phase as a sequence of milestones, clustered For MST collaborations a set of almost generic milestones had been elaborated, allowing for the above mentioned partition of the project in manageable slices (Fig. 5).

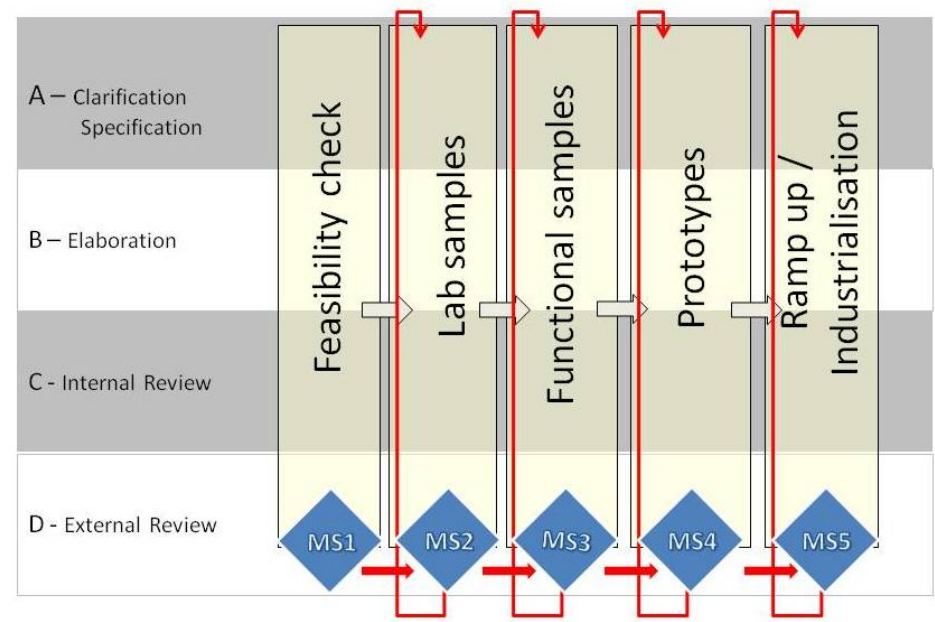

Fig. 4. Framework for the "solution open" MST- Product development 


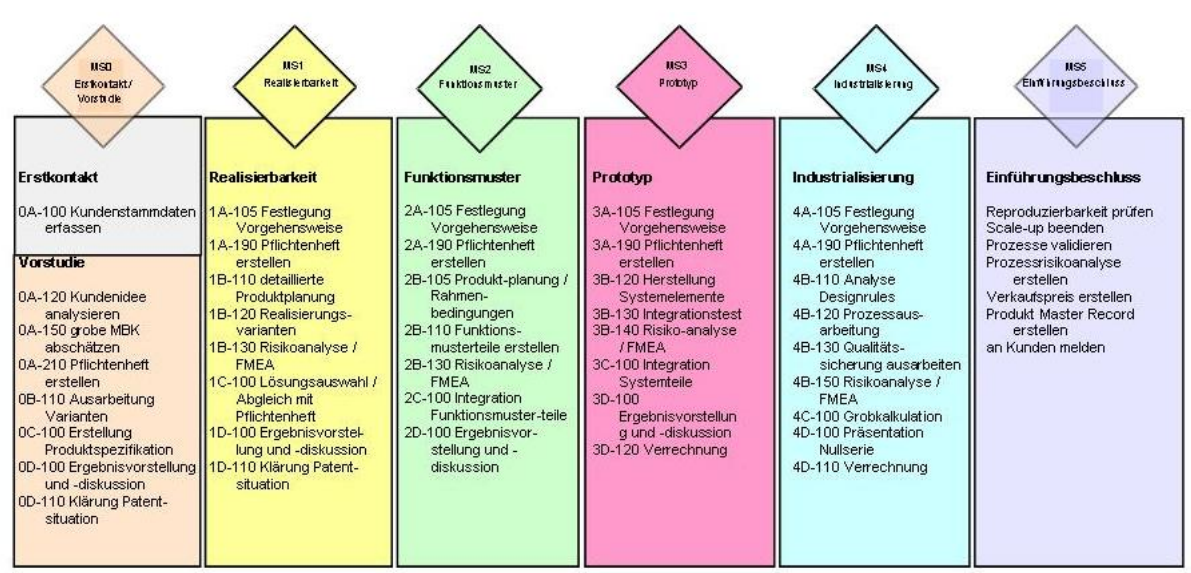

Fig. 5. MST- Design Control and Commercialization Guidelines of the German MicroWebFab Network

This set of milestones, called "Design, Control and Commercialization Guidelines" is organized in a way that supports the iterative processing of the product development.

Each sequence of milestones follows the same logic, introduced by the spiral model. Subtasks dedicated to each single sub-milestone fix the expected outcome of the development process (e.g. FMEA, Patent analysis, etc.). At detailed set of (generic) business process sequences ensures the comprehensive processing of the milestone -related activities. Templates for competences and responsibilities related to each process / milestone allow for a kind of standardized management of the product development process by coordination (Fig. 6).

To support the coordinator and the project team, a set of "control questions" was defined for each sub-milestone. Replying the mandatory and optional questions ensures that a milestone has been processed in a satisfying manner (Fig. 6). The control questions were implemented by web interfaces or offline checklists in Pdf-format.

Using semi-collaborative and ad-hoc workflow-technologies [SCHM99] allowed us to model and to start child processes and also to easily relaunch process sequences with unsatisfying results according to the modified Boehm model. In every phase of a development project the project team or a single person are automatically informed/asked through a "push-" strategy supported through automatically generated emails by the information management systems workflow engine. The emails include the project history, control questions for the related task to fulfill and also consist of hyperlinks to support programs or checklists supporting each single business process. Besides the reminder function for each user the email functionality and the checklists provided for a log-book/ documentation functionality.

The replies to the emails were sent back to the information management systems are used as a feedback mechanism allowing the coordinator to become informed via a management dashboard functionality linked to the integrated database for storage of the operational and technological information. 


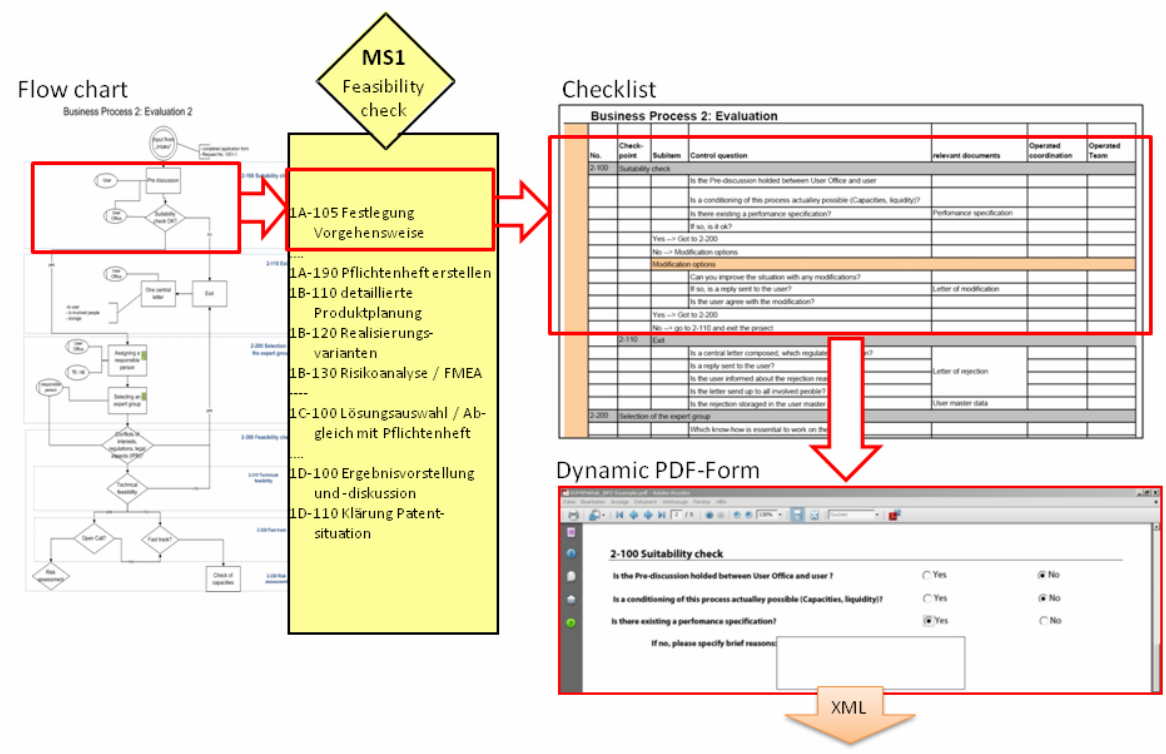

Fig. 6. Derivation of manageable information elements for the solution-open MST product development process

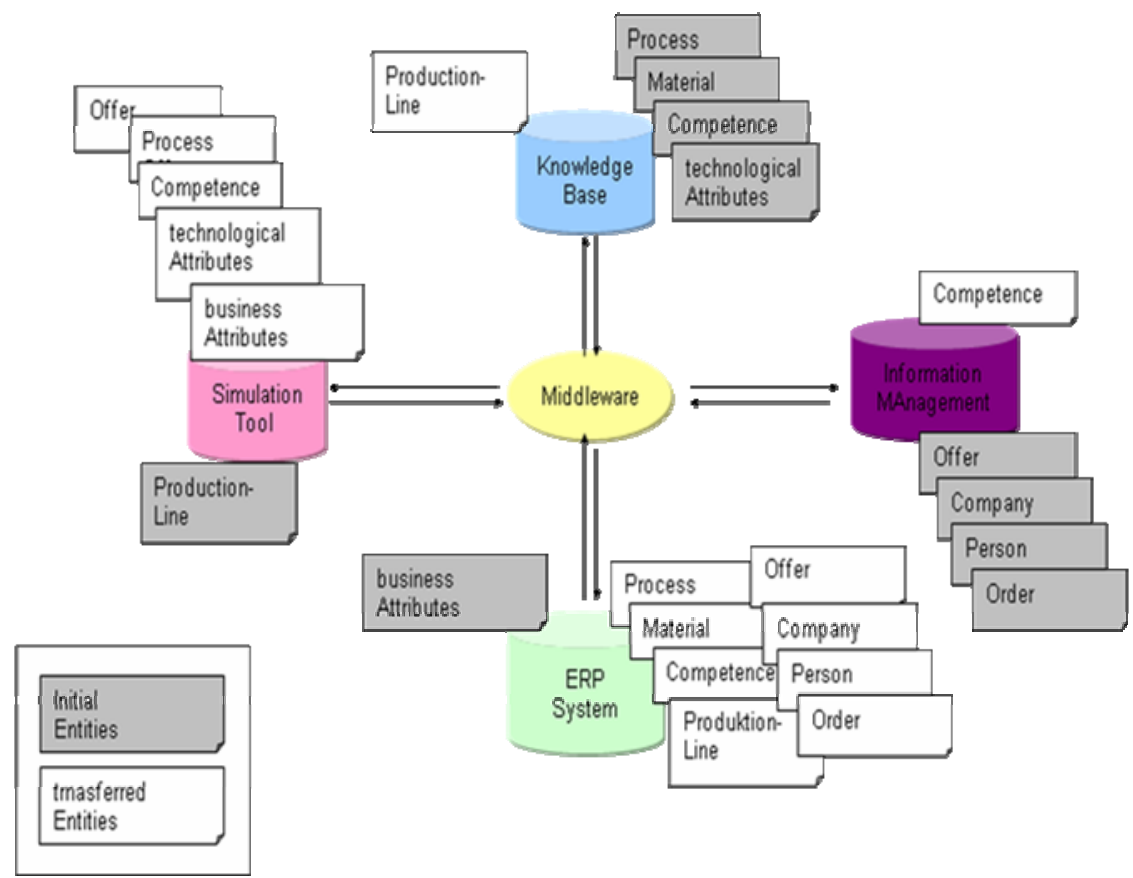

Fig. 7. Enterprise Application Integration Strategies to link SME software solutions in a demand oriented manner 
An Enterprise Application Integration (EAI) solution in the background ensures the information exchange between the heterogeneous applications on server side (Enterprise Resource Planning system, Knowledge database, information system, MESsystems), preventing inconsistency between the different applications. In contrary to other state of the art service architectures (e.g. the SAP net weaver architecture) which are often used in proprietary environments for the provision of standardized services, the EAI-concept focuses more on the provision of dedicated interfaces for data access and data exchange [KAIB02]. Fig. 7 shows an Example for an SME network of seven companies in the field of MST.

The middleware infrastructure works similar to an email post box framework. Information objects are posted in different folders for exchange of information via a XML based information exchange methods. A server side middleware mechanism interprets the incoming information elements translates information in an other data schemes (if necessary). The other client applications frequently poll the postbox folder and catch the information available.

\section{Discussion of the Concept - Experiences Made within a SME - Cooperation in the Field of Micro Technology}

The following chapter summarizes the experiences having been made while the operation of the German MicroWebFab cooperation, a SME network operating in the field of Biotechnology in its seventh year.

\section{Legal Bindings are not mandatory for a successful operation of Micro production networks}

In contrary to first expectations a convincingly managed virtualisation of development and production services is not only accepted by customers that are experienced in the MST market with its foundry services. It is - in the meantime - also accepted by customers from other industries that want to replace their existing technologies by use of (disruptive) microsystems technology. To top it all off MicroWebFab's customers appreciated the business idea as a way to give them access to MST competence and/or to assess MST alternatives against their own approaches on a shoestring. The MicroWebFab business model of a company operating self contained at the market is still the vision of the partners but not longer a prerequisite for success. Due to the fact that it is a long way to full acceptance and to profits the consortium developed a concept called Evolutionary Cooperation Networks [DICK08] was introduced, which gives guard rails for organisation advancement.

\section{Shareholder and stakeholder structure}

One of the basic assumptions of the MicroWebFab solution concept comprised that the participating technological companies are both, share- and stakeholders of the cooperation. The advantage of this concept is that there is a commonly developed concept and business strategy, which shall prevent basic misunderstandings about the basis of the cooperation's contract.

Furthermore it ensures a direct access from and to the company's key processes and persons in the technological and organisational dimension. The proof of this basic 
thesis showed that the structure is one the one hand side a guarantee for proper implementation of the slogan "The sum is greater than its parts". This very strict assignment on the other hand produces certain sensitivity of the cooperation in terms of mutual dependence of partners.

\section{Influence of the ICT infrastructure}

The MicroWebFab example shows quite well, that a state of the art ICT Infrastructure with well defined interfaces on organisational and operational provides for some advantages in the competition with other networks or even larger companies. Effectiveness of communication and an extensive knowledge exchange can speed up the decision processes and with that lead to a kind of "first come, first serve"-effect. Finally internal effects such as the different trust level between the partners or the broad competence portfolio accessible to customers will be the primary drivers for the cooperation success.

\section{Conclusion and Summary}

More customer demand than ever are looking for one stop shop development and production services provided by SME cooperation. The necessary boundary conditions on organisational level can be also adressed by SMEs, as long as these are willing to intensively cooperate in a new quality of intensity and trust. An appropriate approach for this can be the clan cooperation. The modified MST clan cooperation concept of jointly a marketing and project management of equal SME partners is

- $\quad$ suitable for companies that are in the process of entering new markets

- $\quad$ suitable for smaller specialized companies with limited marketing/sales capacities,

- $\quad$ not suitable for companies with a focus on large scale /automotive market

Rigid and strictly structured IT-infrastructures are not an appropriate solution for this type of cooperation. Furthermore a demand oriented infrastructure has to be modular and flexible, where "flexibility" for an SME network in the context of MST has to address two topics: It has to address the specific requirements of a parallel product development and process adaption, which can be solved by a flexible process model based or an iterative development approach having been described in this paper. In addition it has to deal with restrictions in information exchange coming from the heterogeneous Information technology at the partner sites. To overcome these limitations, a demand oriented, EAI based approach supporting a cross company information exchange has been introduced.

\section{References}

[BOEHM88] Boehm, B.W.: A Spiral Model of Software Development and Enhancement. IEEE Computer 21, 61-72 (1988)

[DICK08] Dickerhof, M.: Ein neues Konzept für das bedarfsgerechte Informations- du Wissensmanagement in Unternehmenskooperationen der Multimaterial Mikrosystemtechnik, Disseration, Universitätsverlag Karlsruhe (2008) 
[KAIB02] Kaib, M.: Enterprise Application Integration. DUV wirtschaftsinformatik, Wiesbaden (2002)

[MWF03] Gengenbach, U.: A project to establish a virtual enterprise for distributed development and fabrication of Microsystems. In: Proceedings of the COMS 2003 conference, Ypsilanti (2003)

[PICO93] Picot, A., Scheer, A.W. (eds.): In Organisationsstrukturen der Wirtschaft und ihre Anforderungen an die Informations- und Kommunikationstechnik. Handbuch Informationsmanagement, Aufgaben - Konzepte - Praxislösungen. Gabler-Verlag (1993)

[SCHM99] Schmidt, A., Düpmeier, C., Eggert, H.: WILDFLOW - components for building scientific and technical work environments. In: 1999 Internat. Conf. on WebBased Modelling and Simulation, San Francisco, Calif., January 17-20 (1999)

[VIRT09] N.N., Web portal of the California Network Virtual Enterprise, http: / /www. virtualenterprise.org/ (2009.07.20) 